\title{
Research on Hierarchical Scheduling of Operational Operation of Polar Meteorological Satellite Ground System
}

\author{
Zhaohui Cheng ", Manyun Lin, Xianggang Zhao, You Ma, Cunqun Fan ${ }^{\text {b,* }}$ \\ National Satellite Meteorological Centre, Beijing, China \\ a email:chengzh@cma.gov.cn, ${ }^{b}$ email:fancq@cma.gov.cn \\ *corresponding author
}

\begin{abstract}
Keywords: Meteorological satellite; Ground application system; Hierarchical scheduling; Planning scheduling

Abstract: Meteorological satellite ground application system business operation scheduling is the core of the entire business operation, directly related to the entire system operating efficiency. This paper designs a hierarchical satellite terrestrial system business operation scheduling method. First of all, the satellite service is classified into first-level and secondlevel scheduling. Then, based on the hierarchical scheduling, task scheduling and scheduled scheduling are implemented. Through hierarchical scheduling and planning and scheduling to achieve the entire system job scheduling, thereby enhancing the efficiency of task scheduling and improve the overall system operating efficiency.
\end{abstract}

\section{Introduction}

Each system of polar orbit meteorological satellites needs to be able to collaborate with each other such as receiving, transmitting, processing and archiving. Due to the large size of the satellite system and the large number of operations, it is necessary to rationally schedule the various satellite ground applications.

Research on task scheduling has made some achievements. The paper [1] point out Hu's scheduling algorithm was suggested for solving this task. The proposed approach allows for generation of an optimal execution schedule for versions of the N-version software of the control system of a satellite communications system. The schedule has the advantage of using a reduced time for task solving. The paper proposed a solution to determination of the minimum execution time to run the $\mathrm{N}$-version of the control system of a satellite communications system for a given number of processors. The paper shows a procedure for determination of the number of processors required in the minimum time for execution of a task flow. The paper [2] proposed a novel dynamic fault-tolerant scheduling model for real-time tasks on multiple observation satellites. In this model, the primary/backup policy is employed to tolerate one satellite's permanent failure at one time instant. The paper [3] make conclusive analysis on the relevant researches home and aboard, and brings out prospects for future research. Firstly, the type and description of uncertainties in imaging 
satellite scheduling are analyzed. Secondly, research progress is concluded from three aspects: proactive scheduling, reactive scheduling and proactive-reactive scheduling. Thirdly, some issues existing in the research are analyzed like inexactness of uncertainties estimation methods. The paper [4] considered the characteristic of the problem, a constraint satisfaction problem model with two objective functions was established. Furthermore, a satellite periodic continuous observation task scheduling algorithm based on multiobjective evolutionary algorithm was proposed. The paper [5] proposed a two-phase task scheduling algorithm to enhance the performance of scheduling, including an initial scheduling phase and a dynamic scheduling phase. In the initial scheduling phase, they construct a scheduling model with multiple constraint conditions, and also design an improved Genetic Algorithm (GA) with elite reserved strategy and a crowding function to find the initial scheduling solution.

Based on the above research, this paper designs a hierarchical service scheduling method for satellite terrestrial systems. First of all, the satellite service is classified into first-level and secondlevel scheduling. Then, based on the hierarchical scheduling, task scheduling and scheduled scheduling are implemented.

\section{Scheduling Strategy}

In order to ensure that all systems of the meteorological satellite application system can accomplish various tasks of receiving, transmitting, processing and archiving in coordination with each other. The application system adopts the hierarchical scheduling mode to realize the normal operation of the entire satellite application system.

A scheduling: According to the satellite operating conditions, satellite orbit and satellite ground system status to develop multi-satellite operating schedule, multi-satellite operating schedule or binary scheduling command binary satellite remote sensing data reception, transmission and processing business scheduling tasks;

Secondary scheduling: satellite data receiving system, data preprocessing system, product generation system, product quality inspection system, computer and network system, data archiving and service system, and according to the business operation schedule and the operation of its own business needs to generate the system Business running schedule to schedule the system's business operations.

The core of the ground application system business operation not only needs to schedule all the scheduled tasks needed to complete the operation of the system itself, but also tracks and monitors the operating status of other systems according to the time-of-flight reception schedule of the multisatellite track. According to the different scheduling methods and scheduling conditions, the scheduling software in the design is divided into two kinds: scheduled tasks and scheduled jobs, which form the basis and core of business operations.

\subsection{Scheduled Task Scheduling}

Scheduled task scheduling system to complete the daily affairs management tasks, including multi-orbit forecast, multi-star file transfer, multi-star data archiving, file cleaning and database maintenance and other related work. The schedule of multi-satellite orbit forecast generation is an important basis for planning job scheduling and receiving and scheduling plans made by various ground stations. Scheduled scheduling schedule shown in Figure 1. 


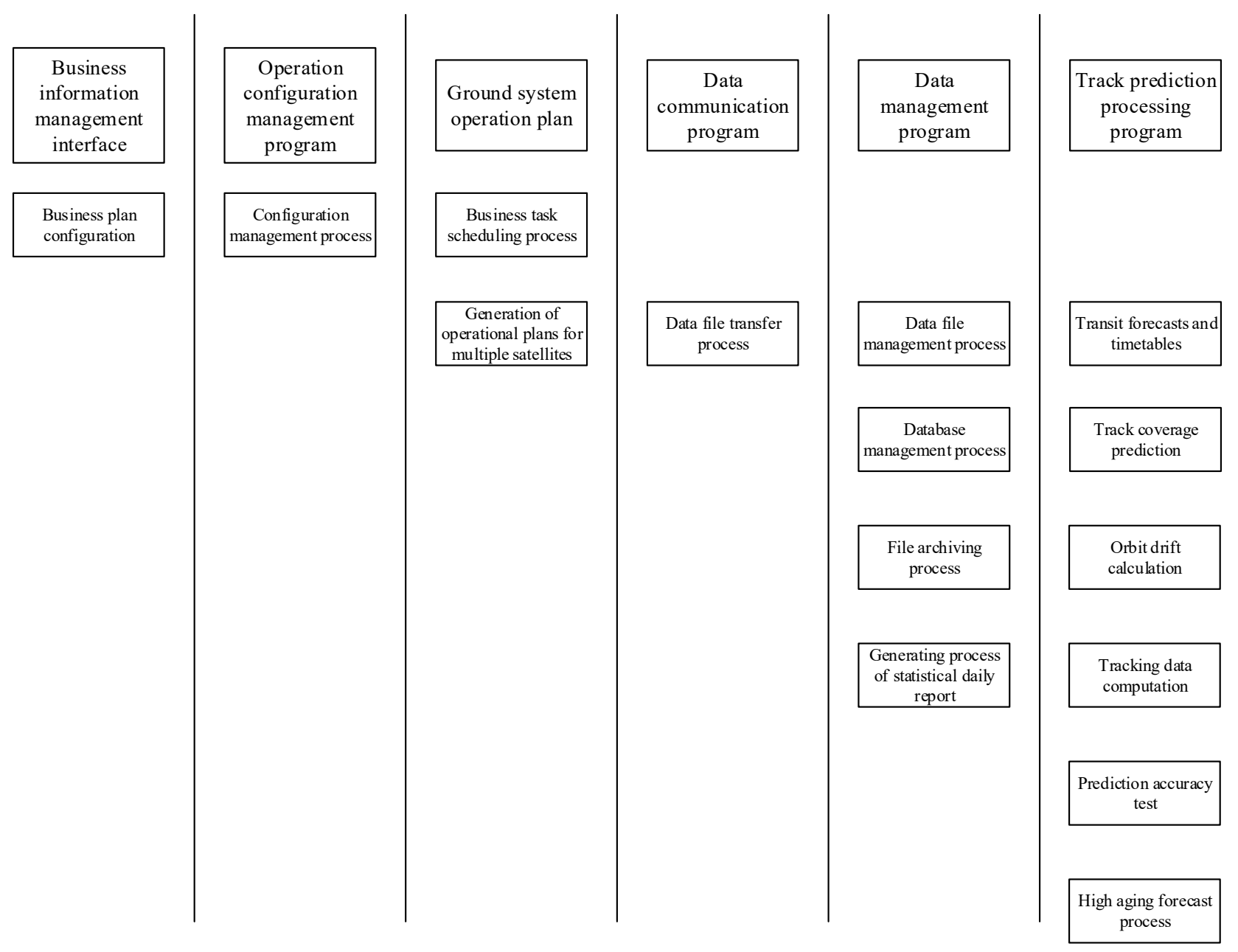

Figure 1 Scheduled scheduling process

\subsection{Scheduled Job Scheduling}

Scheduled job scheduling realizes the tracking and monitoring of real-time transit of binary data of the meteorological satellite ground application system, including the steps of receiving binary data, optimizing splicing, quality inspection and transmission.

Scheduled job scheduling is realized by the real-time reception and transmission tracking process of binary data and the real-time reception and transmission tracking process of multiple satellite data. Each job scheduling process realizes a tracking job flow of a satellite transit track. The currently configured Scheduled Job Scheduler processes data in real time to receive Transport Trace job processes and other satellite data in real time to receive Transport Trace job processes.

In order to coordinate the operation of the entire ground application system, the operation of each system must be fully considered during the design process to ensure the completeness and effectiveness of the scheduling as much as possible. As the controller of the entire ground application system, Relatively complex, induction and design of the interface is an important point of the entire design.

1) Dispatch management automation combines with manual intervention: As the operation management center of the ground application system, the dispatch management of each subsystem must be carried out. Automatically generate the current track transit tracking job plan through the multi-satellite track transit receiving schedule and job configuration templates, and automatically generate the same day regular scheduled tasks and job configuration templates through the task 
configuration template. The task configuration template can be edited and modified by the client software.

2) Solutions for Scheduling Tasks and Working Across Sky Problems: Because internal scheduling has high requirements on time, task scheduling is the most difficult task to solve for scheduling management. For this reason, the scheduling time is counted in seconds after 1970 to make the scheduling time accurate To the second level, while solving the task across the sky.

\section{Orbital Parameters of the Download and Distribution}

Orbit parameter download and distribution Server-side satellite orbit parameter acquisition and processing process, the number of tracks to add and verify the process, the binary satellite orbit and the two lines reported TBUS production process, by the client's satellite orbit fusion monitoring program monitoring.

Satellite orbit parameters acquisition and processing process automatically run daily, from the external network to download all satellite orbit number, and its accuracy testing and format conversion. The track number adding and checking process runs daily to test the server-side orbit data. When finding that the number of FY-3 is not updated, the number of the latest FY-3 is found and the alarm $\log$ is sent. The client's satellite orbit fusion monitoring program can check whether the number of orbits is fused daily to ensure the timely updating of the number of orbits of FY-3.

Satellite orbit transit forecasting and reception schedule generation process is the core process of orbit prediction. Its main function is to calculate the cross-satellite and multi-station transit data according to the satellite receiving mission of the terrestrial system and record the correspondence of each track passing over each station At the same time, the number of transit orbits, lifting track marks, over elevation and azimuth are given, and the time when the satellite passes the obstruction is calculated according to the obstruction identification of the station and the obstructing azimuth, And the receiving time and ending time of multi-station coverage in the same orbit, the starting and ending time of data transmission of each station are given. According to the principle of real-time and delay receiving, the receiving time and transmission time corresponding to the two conditions are calculated respectively.

\section{Conclusions}

Meteorological satellite ground application system business operation scheduling is the core of the entire business operation, directly related to the entire system operating efficiency. This paper designs a hierarchical satellite terrestrial system business operation scheduling method. First of all, the satellite service is classified into first-level and second-level scheduling. Then, based on the hierarchical scheduling, task scheduling and scheduled scheduling are implemented. Through hierarchical scheduling and planning and scheduling to achieve the entire system job scheduling, thereby enhancing the efficiency of task scheduling and improve the overall system operating efficiency.

\section{Acknowledgements}

The work presented in this study is supported by National High-tech R\&D Program (2011AA12A104). 


\section{References}

[1] Chernigovskiy A S, Tsarev R Y, Knyazkov A N. Hu's algorithm application for task scheduling in N-version software for satellite communications control systems[C]// International Siberian Conference on Control and Communications. IEEE, 2015:1-4.

[2] Zhu X M, Wang J J, Wang J, et al. Fault-Tolerance Oriented Real-Time Task Scheduling on Multiple EarthObserving Satellites[J]. Acta Electronica Sinica, 2015.

[3] Li Z, Li X. Current Status and Prospect of Imaging Satellite Task Dynamic Scheduling Methods[C]// International Conference on Intelligent Human-Machine Systems and Cybernetics. IEEE, 2016:436-439.

[4] Chen H, Du C, Li J, et al. An approach of satellite periodic continuous observation task scheduling based on evolutionary computation[C]// Genetic and Evolutionary Computation Conference Companion. ACM, 2017:15-16. [5] Deng B, Jiang C, Kuang L, et al. Two-Phase Task Scheduling in Data Relay Satellite Systems[J]. IEEE Transactions on Vehicular Technology, 2017, PP(99):1-5. 